\title{
On summability, integrability and impulsive differential equations in Banach spaces
}

Seppo V Heikkilä*

To Jean Mawhin on the occasion of his seventieth birthday

"Correspondence: sheikki@cc.oulu.fi Department of Mathematical Sciences, University of Oulu, P.O. Box 3000, Oulu, 90014, Finland

\begin{abstract}
Purpose: To study summability of families indexed by well-ordered sets of $\mathbb{R} \cup\{\infty\}$ in normed spaces. To derive integrability criteria for step mappings and for right regulated mappings from an interval of $\mathbb{R} \cup\{\infty\}$ to a Banach space. To study solvability of impulsive differential equations.

Main methods: A generalized iteration method presented, e.g., (Heikkilä and Lakshmikantham in Monotone Iterative Techniques for Discontinuous Nonlinear Differential Equations, 1994). Summability of families in normed spaces indexed with well-ordered subsets of $\mathbb{R} \cup\{\infty\}$.
\end{abstract}

Results: Necessary and sufficient conditions for global and local HK, HL, Bochner and Riemann integrability of step mappings and right regulated mappings defined on an interval of $\mathbb{R} \cup\{\infty\}$, and having values in a Banach space. Applications to impulsive differential equations are also presented. Families indexed with well-ordered subsets of $\mathbb{R} \cup\{\infty\}$ are used to represent impulsive parts of considered equations and to approximate their solutions.

MSC: 26A06; 26A18; 26A39; 26B12; 26E20; 34A36; 34A37; 34G20; 40A05; 40D05; 40F05; 47H07; 47H10

Keywords: family; well-ordered index set; summable; primitive; integral; step mapping; right regulated; differential equation; impulsive; generalized iteration method

\section{Introduction}

In Chapter VIII of his book, 'Foundations of Modern Analysis', Jean Dieudonné criticized the adoption of the Riemann integral in Calculus courses as follows: 'Only the stubborn conservatism of academic tradition could freeze it into a regular part of curriculum, long after it had outlived its historical importance.' The integral presented in the book is justified as follows: 'to dispense with any considerations drawn from measure theory; this is what we have done by defining only the integral of regulated mappings (sometimes called the 'Cauchy integral'). When one needs a more powerful tool, there is no point in stopping halfway, and the general theory of ('Lebesgue') integral is the only sensible answer.'

On the other hand, a few years before the publication in 1960 of the book [1] cited above, Ralph Henstock and Jaroslav Kurzweil generalized the definition of the Riemann integral so that the resulting integral, called here the Henstock-Kurzweil (shortly HK) integral, encloses the Lebesgue integral of real valued functions. The study of HK integrals of Banach

(c) 2013 Heikkilä; licensee Springer. This is an Open Access article distributed under the terms of the Creative Commons Attribution License (http://creativecommons.org/licenses/by/2.0), which permits unrestricted use, distribution, and reproduction in any medium, provided the original work is properly cited. 
valued mappings started around 1990 by the work of R.A. Gordon. The strong version of HK integral is called here the Henstock-Lebesgue (shortly HL) integral. No measure theory is needed in the definitions of HK and HL integrals. Moreover, if a mapping $g$ from a compact real interval $I$ to a Banach space $E$ is Bochner integrable, i.e., if the norm function $t \mapsto\|g(t)\|$ is Lebesgue integrable, then $g$ is also HL integrable. Converse is not true because the norm function of a HL integrable mapping is not necessarily HL integrable or HK integrable. Moreover HL integrability encloses improper integrals on finite intervals; HK integrability also on unbounded intervals.

In [1], the integral calculus is presented for regulated mappings, i.e., the mappings from a real interval $I$ to a Banach space $E$, having left limits in $I \backslash\{\inf I\}$ and right limits in $I \backslash\{\sup I\}$. The definition of the integral of a mapping $g: I \rightarrow E$ is based on the existence of a primitive, i.e., a continuous mapping $f: I \rightarrow E$ that is differentiable in the complement of a countable subset $Z$ of $I$, and $f^{\prime}(t)=g(t)$, for each $t \in I \backslash Z$. Because any two primitives of $g$ differ by a constant, the difference $f(b)-f(a)$ for any two points of $I$, is independent of the particular primitive $f$. This difference is written $\int_{a}^{b} g(t) d t$, and is called the integral of $g$ from $a$ to $b$. As shown in [1], a primitive exists for every regulated mapping.

In this paper, we study integrability of right regulated mappings, i.e., those mappings from an interval $I$ of $\mathbb{R} \cup\{\infty\}$ to a Banach space $E$, which have right limits at every point of $I \backslash\{\sup I\}$. The main difference between regulated mappings and right regulated mappings is that the latter ones may have discontinuities of the second kind, while regulated mappings can have only discontinuities of the first kind. Another difference is that regulated mappings are HL integrable and Riemann integrable on bounded intervals, whereas all right regulated mappings are not even $\mathrm{HK}$ integrable. The main purpose of this paper is to develop criteria for $\mathrm{HK}, \mathrm{HL}$, Bochner and Riemann integrability of right regulated mappings on an interval $I$ of $\mathbb{R} \cup\{\infty\}$. Necessary and sufficient conditions for local integrability are also presented. The main tools are:

- A generalized iteration method presented, e.g., in [2]. Using this method, we shall prove that a right regulated mapping has at most countable number of discontinuities, and that it can be approximated uniformly on compact intervals by step mappings with well-ordered steps. A fixed-point theorem based on this method is applied in the study of impulsive differential equations.

- Summability of families in normed spaces. Summability of families with nonempty index sets is studied, e.g., in [1, 3, 4]. The given definitions rule out conditional summability, so that the obtained summability results are not applicable in the study of HK and HL integrability. Therefore, we assume that the index set is well ordered. For the sake of applications, we assume that the index set is contained in $\mathbb{R} \cup\{\infty\}$.

- CD primitives. By a CD primitive of a mapping $g$ from an interval $I$ of $\mathbb{R} \cup\{\infty\}$ to $E$ we mean a continuous mapping $f: I \backslash \sup I \rightarrow \mathbb{R}$ that is differentiable in the complement of a countable subset $Z$ of $I$, and $f^{\prime}(t)=g(t)$ for each $t \in I \backslash Z$.

This paper is organized as follows. In Section 2, we define and study summability and absolute summability of a family $\left(x_{\alpha}\right)_{\alpha \in \Lambda}$ in a normed space when the index set $\Lambda$ is a well ordered subset of $\mathbb{R} \cup\{\infty\}$. With the help of such families, we derive necessary and sufficient conditions for global and local HK, HL, Bochner and Riemann integrability of step mappings and right regulated mappings defined on an interval of $\mathbb{R} \cup\{\infty\}$, and having values in a Banach space. The results obtained for step mappings in Section 3 both generalize and improve some results derived in [5-7] (see Remark 3.1). The integrability criteria de- 
rived in Section 4 for right regulated mappings are new. We shall prove, for instance, the following results for a right regulated mapping $g: I \rightarrow E,-\infty<\min I<\sup I \leq \infty$. (We say that a property holds locally for a function defined on $I$, if the function has that property on every compact subinterval of $I$.)

1. $g$ is locally HL integrable if and only if it has a CD primitive.

2. $g$ is HL integrable when $I$ is bounded if and only if $g$ has a CD primitive that has the left limit at $\sup I$.

3. $g$ is HK integrable if it has a CD primitive that has the left limit at $\sup I$.

4. $g$ is locally Bochner integrable if and only if it has a locally absolutely continuous CD primitive.

5. $g$ is Bochner integrable if and only if it has a locally absolutely continuous CD primitive that has the left limit at $\sup I$.

6. $g$ is locally Riemann integrable if and only if it is locally bounded, in which case $g$ has a locally Lipschitz continuous CD primitive.

7. $g$ is Riemann integrable if and only if it is bounded and $I$ is bounded.

8. The improper Riemann integral of $g$ from $\min I$ to $\sup I$ exists if $g$ is locally bounded, and its CD primitive has the left limit at $\sup I$.

9. For each compact subinterval $[a, b]$ of $I$, either $g$ is Riemann integrable on $[a, b]$, or there exists the greatest number $c_{1}$ in $(a, b]$ such that $g$ is locally Riemann integrable on $\left[a, c_{1}\right)$.

10. For each compact subinterval $[a, b]$ of $I$, either $g$ is Bochner integrable on $[a, b]$, or there exists the greatest number $c_{2}$ in $(a, b]$ such that $g$ is locally Bochner integrable on $\left[a, c_{2}\right)$.

11. For each compact subinterval $[a, b]$ of $I$, either $g$ is HL integrable on $[a, b]$, or there exists the greatest number $c_{3}$ in $(a, b]$ such that $g$ is locally HL integrable on $\left[a, c_{3}\right)$.

Concrete examples of mappings $f, g: \mathbb{R}_{+} \rightarrow E$ are presented for above results when $E$ is the space $c_{0}$ of those sequences of real numbers which converge to 0 . In every example, the mapping $g$ has the discontinuity of second kind at every rational point of $\mathbb{R}_{+}$. The above results are valid with minor modifications also when $g$ is left regulated, i.e., when $g$ has left limits at every point of $I \backslash\{\inf I\}$.

The first one of the above results will be applied in Section 5 to impulsive differential equations in Banach spaces. Families indexed with well ordered subsets of $\mathbb{R} \cup\{\infty\}$ are used to represent impulsive parts of considered equations and to approximate their solutions.

\section{Preliminaries}

In this section, we shall first present basic properties of well-ordered subsets of $\mathbb{R} \cup\{\infty\}$. These sets are used as index sets of families in normed spaces. After defining summability and presenting examples of such families we introduce basic facts on HK, HL, Bochner and Riemann integrability of mappings from a real interval to a Banach space.

A nonempty subset $\Lambda$ of $\mathbb{R} \cup\{\infty\}$, ordered by the natural ordering $<$ of $\mathbb{R}$, plus $t<\infty$ for every $t \in \mathbb{R}$, is well ordered if every nonempty subset of $\Lambda$ has the smallest element. In particular, to every number $\beta$ of $\Lambda$, different from its possible maximum, there corresponds the smallest element in $\Lambda$ that is greater than $\beta$. It is called the successor of $\beta$ and is denoted by $S(\beta)$. There are no numbers of $\Lambda$ in the open interval $(\beta, S(\beta))$. The following properties are needed: 
- Every well-ordered subset of $\mathbb{R} \cup\{\infty\}$ is countable.

- Principle of Transfinite Induction: If $\Lambda$ is well ordered and $\mathcal{P}$ is a property such that if $\mathcal{P}(\gamma)$ is true whenever $\mathcal{P}(\beta)$ is true for all $\beta<\gamma$ in $\Lambda$, then $\mathcal{P}(\gamma)$ is true of all $\gamma \in \Lambda$.

Definition 2.1 Let $\Lambda$ be a well-ordered subset of $\mathbb{R} \cup\{\infty\}$. Denote $a=\min \Lambda$, and $b=$ $\sup \Lambda$. When $\gamma \in \Lambda \cup\{b\}$, denote $\Lambda^{<\gamma}=\{\alpha \in \Lambda \mid \alpha<\gamma\}$. The family $\left(x_{\alpha}\right)_{\alpha \in \Lambda}$ with elements $x_{\alpha}$ in a normed space $E$ is summable if it has the following properties:

(s) To every $\gamma \in \Lambda \cup\{b\}$ there corresponds a unique element $\sigma(\gamma)$ of $E$, called the sum of the family $\left(x_{\alpha}\right)_{\alpha \in \Lambda^{<\gamma}}$, satisfying the following conditions:

(i) $\sigma(a)=0$, and if $\gamma=S(\beta)$ for some $\beta \in \Lambda$, then $\sigma(\gamma)=\sigma(\beta)+x_{\beta}$.

(ii) If $\gamma$ is not a successor, then for each $\epsilon>0$ there is such $\beta_{\epsilon} \in \Lambda^{<\gamma}$ that $\|\sigma(\beta)-\sigma(\gamma)\|<\epsilon$ whenever $\beta \in \Lambda$ and $\beta_{\epsilon} \leq \beta<\gamma$.

The sum $\sigma$ of a summable family $\left(x_{\alpha}\right)_{\alpha \in \Lambda}$ is $\sigma(b)$ if $b \notin \Lambda$, and $\sigma(b)+x_{b}$ if $b \in \Lambda$. If $\sigma(\gamma)$ is defined for every $\gamma \in \Lambda$, we say that $\left(x_{\alpha}\right)_{\alpha \in \Lambda}$ is locally summable. A family $\left(x_{\alpha}\right)_{\alpha \in \Lambda}$ is (locally) absolutely summable if $\left(\left\|x_{\alpha}\right\|\right)_{\alpha \in \Lambda}$ is (locally) summable.

Remarks 2.1 The above definition of summability is analogous to that given in [8] when the index set $\Lambda$ is an ordinal. Because $\Lambda$ is countable, the given definition of absolute summability of a family $\left(x_{\alpha}\right)_{\alpha \in \Lambda}$ agrees on that of [1, Section V.3], i.e., for a bijection $\varphi$ from $\mathbb{N}$ to $\Lambda$ the series $\sum_{n=1}^{\infty} x_{\varphi(n)}$ is absolutely convergent. As for results dealing with ordinary, unconditional and absolute convergence of $\sum_{n=0}^{\infty} z_{n}$ when $E$ is a Banach space; see, e.g., [6, Appendix B].

Next, we shall determine the first partial sums and the sum of a summable family $\left(x_{\alpha}\right)_{\alpha \in \Lambda}$ in some elementary cases ( $\sup _{\Lambda}$ means the least upper bound in $\Lambda$ ).

1. If $\Lambda$ is finite and nonempty, then $\Lambda=\left\{S^{n}(a) \mid n=0, \ldots, m\right\}, m \in \mathbb{N}_{0}\left(S^{0}(a)=a\right)$.

2. If $\Lambda=\left\{S^{n}(a) \mid n \in \mathbb{N}_{0}\right\}$, then $\sigma=\sum_{n=0}^{\infty} x_{S^{n}(a)}=\sum_{n=0}^{\infty} z_{n}$, where $z_{n}=x_{S^{n}(a)}, n \in \mathbb{N}_{0}$.

3. After $S^{n}(a), n \in \mathbb{N}_{0}$, the next possible numbers of $\Lambda$ are $a_{0}=\sup _{\Lambda}\left\{S^{n}(a) \mid n \in\right.$ $\left.\mathbb{N}_{0}\right\}, S^{m}\left(a_{0}\right), m=0,1, \ldots, a_{1}=\sup _{\Lambda}\left\{S^{m}\left(a_{0}\right) \mid n \in \mathbb{N}_{0}\right\}, \ldots, S^{m}\left(a_{i}\right), m=0,1, \ldots, a_{i+1}=$ $\sup _{\Lambda}\left\{S^{m}\left(a_{i}\right) \mid n \in \mathbb{N}_{0}\right\}, i=0,1, \ldots, b_{0}=\sup _{\Lambda}\left\{a_{i} \mid i \in \mathbb{N}_{0}\right\}$, and so on. Corresponding partial sums of the family $\left(x_{\alpha}\right)_{\alpha \in \Lambda}$ are:

$$
\begin{aligned}
& \sigma\left(S^{m}\left(a_{0}\right)\right)=\sigma\left(a_{0}\right)+\sum_{n=0}^{m-1} x_{S^{n}\left(a_{0}\right)}=\sum_{n=0}^{\infty} x_{S^{n}(a)}+\sum_{n=0}^{m-1} x_{S^{n}\left(a_{0}\right)}, \\
& \sigma\left(a_{1}\right)=\sigma\left(a_{0}\right)+\lim _{m \rightarrow \infty} \sum_{n=0}^{m-1} x_{S^{n}\left(a_{0}\right)}=\sum_{n=0}^{\infty} x_{S^{n}(a)}+\sum_{n=0}^{\infty} x_{S^{n}\left(a_{0}\right)}, \\
& \sigma\left(a_{i+1}\right)=\sigma\left(a_{i}\right)+\lim _{m \rightarrow \infty} \sum_{n=0}^{m-1} x_{S^{n}\left(a_{i}\right)}=\sum_{n=0}^{\infty} x_{S^{n}(a)}+\sum_{j=0}^{i}\left(\sum_{n=0}^{\infty} x_{S^{n}\left(a_{j}\right)}\right), \\
& \sigma\left(b_{0}\right)=\sum_{i=0}^{\infty} \sigma\left(a_{i}\right)=\sum_{n=0}^{\infty} x_{S^{n}(a)}+\sum_{j=0}^{\infty}\left(\sum_{n=0}^{\infty} x_{S^{n}\left(a_{j}\right)}\right)
\end{aligned}
$$

and so on. In particular, if $b=\sup \Lambda=b_{0}$, we have the associative rule: $\sigma(b)=\sum_{i=0}^{\infty} \sigma\left(a_{i}\right)$, where the sum of $\left(x_{\alpha}\right)_{\alpha \in \Lambda^{<b}}$ is presented as a sum of an infinite number of its partial sums. However, this presentation is not independent on the order of both partial sums and their elements, as in the case of absolutely or unconditionally summable families. 
Example 2.1 A simple example of a well-ordered subset of an interval $[a, b)$ of $\mathbb{R}$ is an increasing sequence formed by numbers

$$
b-2^{-n}(b-a), \quad n \in \mathbb{N}_{0} .
$$

The smallest number of this sequence is $a$ and its supremum is $b$. When $a=0$ and $b=1$, we obtain the sequence

$$
\Lambda_{0}=\left\{\alpha\left(n_{0}\right)=1-2^{-n_{0}} \mid n_{0} \in \mathbb{N}_{0}\right\}
$$

The points of $\Lambda_{0}$ divide the interval [0,1) into disjoint subintervals $\left[1-2^{-n_{0}}, 1-2^{-n_{0}-1}\right.$ ), $n_{0} \in \mathbb{N}_{0}$. Choosing $a=1-2^{-n_{0}}, b=1-2^{-n_{0}-1}$ and $n=n_{1}$ in (2.1) we obtain in each of these subintervals decreasing sequences, which together form an inversely well ordered set

$$
\Lambda_{1}=\left\{\alpha\left(n_{0}, n_{1}\right)=1-2^{-n_{0}-1}-2^{-n_{0}-n_{1}-1} \mid n_{0}, n_{1} \in \mathbb{N}_{0}\right\} .
$$

Choosing a vector $e \neq 0$ of $E$ and denoting

$$
x_{\alpha\left(n_{0}, n_{1}\right)}=\frac{(-1)^{n_{1}} 2^{-n_{0}} e}{n_{1}}, \quad n_{0}, n_{1} \in \mathbb{N}_{0}
$$

we obtain a summable family

$$
\left(x_{\alpha\left(n_{0}, n_{1}\right)}\right)_{\alpha\left(n_{0}, n_{1}\right) \in \Lambda_{1}}=\sum_{n_{0}=0}^{\infty} 2^{-n_{0}}\left(\sum_{n_{1}=0}^{\infty} \frac{(-1)^{n_{1}} e}{n_{1}}\right) .
$$

The above process can be continued in the obvious way. For each $m \in \mathbb{N}_{0}$ one obtains a well-ordered set

$$
\Lambda_{m}=\left\{\alpha\left(n_{0}, \ldots, n_{m}\right)=1-\sum_{k=0}^{m-1} 2^{-\sum_{j=0}^{k} n_{j}-j-1}-2^{-\sum_{j=0}^{m} n_{j}-m} \mid n_{0}, \ldots, n_{m} \in \mathbb{N}_{0}\right\} .
$$

Denoting

$$
x_{\alpha\left(n_{0}, \ldots, n_{m}\right)}=\frac{(-1)^{n_{m}} 2^{-\sum_{k=0}^{m-1} n_{k}} e}{\sqrt[m]{n_{m}}}, \quad n_{0}, \ldots, n_{m} \in \mathbb{N}_{0},
$$

then the family

$$
\left(x_{\alpha\left(n_{0}, \ldots, n_{m}\right)}\right)_{\alpha\left(n_{0}, \ldots, n_{m}\right) \in \Lambda_{m}}=\sum_{n_{0}=0}^{\infty}\left(\ldots\left(\sum_{n_{m-1}=0}^{\infty}\left(\sum_{n_{m}=0}^{\infty} \frac{(-1)^{n_{m}} 2^{-\sum_{k=0}^{m-1} n_{k}} e}{\sqrt[m]{n_{m}}}\right)\right)\right)
$$

is summable but neither absolutely nor unconditionally summable.

In the above considerations, $\min \Lambda_{m}=0 \sup \Lambda_{m}=1$ and for every $m \in \mathbb{N}_{0}$. Another way is to restrict $\Lambda_{0}$ to $\left[0, \frac{1}{2}\right), \Lambda_{1}$ to $\left[\frac{1}{2}, \frac{3}{4}\right)$, and in general, restrict $\Lambda_{m}$ to $\left[1-\frac{1}{2^{m}}, 1-\frac{1}{2^{m+1}}\right)$, $m \in \mathbb{N}_{0}$. Thus, $\alpha\left(n_{0}, \ldots, n_{m}\right)$ is replaced by $\beta\left(n_{0}, \ldots, n_{m}\right)=2^{-m-1}\left(1+\alpha\left(n_{0}, \ldots, n_{m}\right)\right)$, i.e.,

$$
\beta\left(n_{0}, \ldots, n_{m}\right)=1-2^{-m-1}-\sum_{k=0}^{m-1} 2^{-\sum_{j=0}^{k} n_{j}-j-m-2}+2^{-\sum_{j=0}^{m} n_{j}-2 m-1} .
$$


These numbers form a well-ordered set

$$
\Lambda_{0}^{1}=\left\{\beta\left(n_{0}, \ldots, n_{m}\right) \mid m, n_{0}, \ldots, n_{m} \in \mathbb{N}_{0}\right\}
$$

satisfying $\min \Lambda_{0}^{1}=0$, and $\sup \Lambda_{0}^{1}=1$.

Replacing in the above considerations $\Lambda_{0}$ by $\Lambda_{0}^{1}$ we obtain more general well-ordered sets of rational numbers: $\Lambda_{m}^{1}, m \in \mathbb{N}_{0}, \Lambda_{0}^{2}, \ldots, \Lambda_{0}^{n}, n \in \mathbb{N}_{0}$. For $n>1$, a family $\left(x_{\alpha}\right)_{\alpha \in \Lambda_{0}^{n}}$ is no more representable as a multiple series.

The following result is needed in the integrability studies.

Lemma 2.1 Let $\left(x_{\alpha}\right)_{\alpha \in \Lambda}$ be a family in $E$ having a well ordered index set $\Lambda$ in $\mathbb{R} \cup\{\infty\}$.

(a) Either $\left(x_{\alpha}\right)_{\alpha \in \Lambda}$ is bounded, or there is the greatest element $c_{1}$ in $\Lambda \backslash\{\min \Lambda\}$ such that the family $\left(x_{\alpha}\right)_{\alpha \in \Lambda<\gamma}$ is bounded for each $\gamma \in \Lambda^{<c_{1}}$.

(b) Either $\left(x_{\alpha}\right)_{\alpha \in \Lambda}$ is absolutely summable, or there is the greatest element $c_{2}$ in $\Lambda \backslash\{\min \Lambda\}$ such that the family $\left(x_{\alpha}\right)_{\alpha \in \Lambda^{<\gamma}}$ is absolutely summable for each $\gamma \in \Lambda^{<c_{2}}$.

(c) Either $\left(x_{\alpha}\right)_{\alpha \in \Lambda}$ is summable, or there is the greatest element $c_{3}$ in $\Lambda \backslash\{\min \Lambda\}$ such that the family $\left(x_{\alpha}\right)_{\alpha \in \Lambda}<\gamma$ is summable for each $\gamma \in \Lambda^{<c_{3}}$.

(d) $c_{1}, c_{2}$ and $c_{3}$ are not successors.

Proof (a) If $\left(x_{\alpha}\right)_{\alpha \in \Lambda}$ is not bounded, there is at least one number $c$ in $\Lambda$ such that $\left(x_{\alpha}\right)_{\alpha \in \Lambda^{<c}}$ is not bounded. Because $\Lambda$ is well ordered, there is the smallest of such numbers $c$. Denoting it by $c_{1}$, then the family $\left(x_{\alpha}\right)_{\alpha \in \Lambda^{<\gamma}}$ is bounded for each $\gamma \in \Lambda^{<c_{1}}$, but not for each $\gamma \in \Lambda^{<c}$, if $c_{1}<c \in \Lambda$. This proves (a).

(b) Assume that the family $\left(x_{\alpha}\right)_{\alpha \in \Lambda}$ is not absolutely summable. Then there is at least one number $c$ in $\Lambda$ such that $\left(x_{\alpha}\right)_{\alpha \in \Lambda^{<c}}$ is not absolutely summable. Because $\Lambda$ is well ordered, there is the smallest of such numbers $c$. Denoting it by $c_{2}$, then the family $\left(x_{\alpha}\right)_{\alpha \in \Lambda^{<\gamma}}$ is absolutely summable for each $\gamma \in \Lambda^{<c_{2}}$, but not for each $\gamma \in \Lambda^{<c}$, if $c_{2}<c \in \Lambda$. This proves (b).

(c) The proof of (c) is similar to that of (b) when absolute summability is replaced by summability.

(d) To prove that $c_{1}$ is not a successor, assume on the contrary that $c_{1}=S(c)$ for some $c \in \Lambda$. Thus $\left(x_{\alpha}\right)_{\alpha \in \Lambda^{<c_{1}}}=\left(x_{\alpha}\right)_{\alpha \in \Lambda^{<c}} \cup\left\{x_{c}\right\}$. Because $\left(x_{\alpha}\right)_{\alpha \in \Lambda^{<c}}$ is unbounded, then $\left(x_{\alpha}\right)_{\alpha \in \Lambda^{<c}}$ also unbounded. But $c<S(c)=c_{1}$, whence $c_{1}$ is not the smallest of those numbers $c$ of $\Lambda$ for which $\left(x_{\alpha}\right)_{\alpha \in \Lambda^{<c}}$ is unbounded, contradicting with the choice of $c_{1}$.

The proofs that $c_{2}$ and $c_{3}$ are not successors are similar.

A mapping $g$ from a compact real interval $[a, b]$ to a Banach space $E$ is called HenstockLebesgue (shortly HL) integrable if there is a mapping $f:[a, b] \rightarrow E$, called a primitive of $g$, with the following property: To each $\epsilon>0$ there corresponds such a mapping $\delta:[a, b] \rightarrow$ $(0, \infty)$ that whenever $[a, b]=\bigcup_{i=1}^{m}\left[t_{i-1}, t_{i}\right]$ and $\xi_{i} \in\left[t_{i-1}, t_{i}\right] \subset\left(\xi_{i}-\delta\left(\xi_{i}\right), \xi_{i}+\delta\left(\xi_{i}\right)\right)$ for all $i=1, \ldots, m$, then

$$
\sum_{i=1}^{m}\left\|f\left(t_{i}\right)-f\left(t_{i-1}\right)-g\left(\xi_{i}\right)\left(t_{i}-t_{i-1}\right)\right\|<\epsilon .
$$


$g$ is called Henstock-Kurzweil (shortly HK) integrable if the above property holds with (2.3) replaced by

$$
\left\|\sum_{i}\left(f\left(t_{i}\right)-f\left(t_{i-1}\right)-g\left(\xi_{i}\right)\left(t_{i}-t_{i-1}\right)\right)\right\|<\epsilon .
$$

Primitives of HK and HL integrable mappings are continuous (see [6, Theorem 7.4.1]). If $g$ is HL (resp. HK) integrable on $[a, b]$, it is HL (resp. HK) integrable on every closed subinterval $[c, d]$ of $[a, b]$. Because any two primitives of $g$ differ by a constant, the difference $f(c)-f(c)$ for any two points of $[a, b]$, is independent of the particular primitive $f$. This difference is called the Henstock-Kurzweil integral of $g$ from $c$ to $d$, and is denoted by $\int_{c}^{d} g(s) d s$. Thus,

$$
\int_{c}^{d} g(s) d s:=f(d)-f(c), \quad \text { where } f \text { is a primitive of } g .
$$

Riemann integrability is obtained when in the definition of HK integrability the gauge functions $\delta$ are replaced by positive constants $\delta$. In this case the integral, defined by (2.4), is called the Riemann integral.

As for the proofs of the following results, see, e.g., [9, Proposition 24.44 and Theorem 24.45].

Lemma 2.2 A mapping $g:[a, b] \rightarrow E$ is Riemann integrable if $g$ is bounded, and is continuous in the complement of a subset $Z$ of $[a, b]$ that has Lebesgue measure 0 . Conversely, every Riemann integrable mapping is bounded.

A mapping $g: I \rightarrow E,-\infty<\min I<\sup I \leq \infty$, is said to be locally integrable in HK, $\mathrm{HL}$, Bochner or Riemann sense if $g$ is HK, HL, Bochner or Riemann integrable on every compact subinterval of $I$.

The next lemma follows, e.g., from [10, Lemma 1.12].

Lemma 2.3 If a mapping $g: I \rightarrow E$ has a CD primitive $f$, then $g$ is locally HL integrable, and (2.4) holds for every compact subinterval $[c, d]$ of $I$.

As for the definition of the HK integral on unbounded real intervals, and the proof of the next result, see [11].

Lemma 2.4 If $-\infty<a<b \leq \infty$ and $g:[a, b] \rightarrow E$, then the following results are equivalent.

(a) $g$ is HK integrable on $[a, c]$ for each $c \in[a, b)$, and $\lim _{c \rightarrow b-} \int_{a}^{c} g(s) d$ s exist.

(b) $g$ is HK integrable on $[a, b]$, and $\int_{a}^{b} g(s) d s=\lim _{c \rightarrow b-} \int_{a}^{c} g(s) d s$.

Remarks 2.2 By definition every HL integrable mapping is HK integrable. Converse holds if $E$ is finite dimensional (see [6, Proposition 3.6.6]). If $b<\infty$, the result of Lemma 2.4 holds when HK integrability is replaced by HL integrability.

A strongly measurable mapping $g:[a, b] \rightarrow E$ is Bochner integrable if and only if the function $t \mapsto\|g(t)\|$ is Lebesgue integrable. Every Bochner integrable mapping is HL integrable. In particular, HL integrability encompasses improper integrals of Bochner integrable mappings. 
Regulated mappings are HK, HL, Bochner and Riemann integrable.

In the proof of the following lemma, we apply a generalized iteration method.

Lemma 2.5 Let $g:[a, b] \rightarrow E$ be right regulated. Then to every positive number $\epsilon$ there corresponds such a well ordered set $\Lambda_{\epsilon}$ in $[a, b]$ that $[a, b)$ is a disjoint union of half-open intervals $[\beta, S(\beta)), \beta \in \Lambda_{\epsilon}^{<b}$, and $\|g(s)-g(t)\| \leq \epsilon$ whenever $s, t \in(\beta, S(\beta))$ and $\beta \in \Lambda_{\epsilon}^{<b}$.

Proof Define $G_{\epsilon}:[a, b] \rightarrow[a, b]$ by $G_{\epsilon}(b)=b$, and

$$
G_{\epsilon}(x)=\sup \{y \in(x, b] \mid\|g(s)-g(t)\| \leq \epsilon \text { for all } s, t \in(x, y)\}, \quad x \in[a, b) .
$$

It is easy to verify that $G_{\epsilon}$ is increasing, i.e., $G_{\epsilon}(x) \leq G_{\epsilon}(y)$ whenever $a \leq x \leq y \leq b$. Because $g$ is right regulated, then $x<G_{\epsilon}(x)$ for each $x \in[a, b)$. By [2, Theorem 1.1.1] there is exactly one well-ordered subset $\Lambda_{\epsilon}$ of $[a, b]$ having the following property:

$$
a=\min \Lambda_{\epsilon} \text { and } a<\gamma \in \Lambda_{\epsilon} \quad \text { if and only if } \quad \gamma=\sup \left\{G_{\epsilon}\left[\left\{\beta \in \Lambda_{\epsilon} \mid \beta<\gamma\right\}\right]\right\} .
$$

Because $\sup G_{\epsilon}\left[\Lambda_{\epsilon}\right]$ exists, it is by [2, Theorem 1.1.1] both a fixed point of $G_{\epsilon}$ and $\max \Lambda_{\epsilon}$. Since $b$ is the only fixed point of $G_{\epsilon}$, then $b=\max \Lambda_{\epsilon}$. Since $\beta<G_{\epsilon}(\beta)$ for each $\beta \in \Lambda_{\epsilon}^{<b}$, it follows from [2, Lemma 1.1.3] that $G_{\epsilon}(\beta)=S(\beta)$ for all $\beta \in \Lambda_{\epsilon}^{<b}$. Thus, by [2, Corollary 1.1.1], $[a, b)$ is the disjoint union of half-open intervals $[\beta, S(\beta)), \beta \in \Lambda_{\epsilon}^{<b}$. The last conclusion follows from (2.5), since $G_{\epsilon}(\beta)=S(\beta)$ for all $\beta \in \Lambda_{\epsilon}^{<b}$.

With the help of Lemma 2.5, we shall prove some properties for right regulated mappings.

Lemma 2.6 Let $g:[a, b] \rightarrow$ E be right regulated. Then

(a) $g$ has at most a countable number of discontinuities.

(b) $g$ is strongly measurable.

Proof (a) Let $\Lambda_{n}, n \in \mathbb{N}$, denote the well ordered subset $\Lambda_{\epsilon}$ defined by (2.6) when $\epsilon=\frac{1}{n}$. It follows from Lemma 2.5 that $\|g(s)-g(t)\| \leq \frac{1}{n}$ whenever $s, t \in(\beta, S(\beta))$ and $\beta \in \Lambda_{n}^{<b}$. Thus all the discontinuity points of $g$ belong to the countable set $Z=\bigcup_{m=1}^{\infty} \Lambda_{m}$.

(b) By (a) the set $Z$ of discontinuity points of $g$ is a null set, whence $g$ is strongly measurable.

\section{On HL, HK, Bochner and Riemann integrability of step mappings}

Let $E$ be a Banach space. In this section, we consider first the integrability of a step mapping $g:[a, b] \rightarrow E,-\infty<a<b<\infty$, that has well ordered steps, i.e., there is a well-ordered subset $\Lambda$ of $[a, b]$ such that $\min \Lambda=a$ and $\max \Lambda=b$, and a family $\left(z_{\alpha}\right)_{\alpha \in \Lambda}$ of $E$ such that

$$
g(t)=z_{\alpha}, \quad t \in[\alpha, S(\alpha)), \alpha \in \Lambda^{<b}
$$

Assume also that $[a, b)$ is a countable union of disjoint intervals $[\alpha, S(\alpha)), \alpha \in \Lambda$. Thus, $g$ is well-defined on $[a, b)$ by $(3.1)$.

As an application of Lemma 2.3, we shall prove the following result. 
Proposition 3.1 Assume that $g:[a, b] \rightarrow E,-\infty<a<b<\infty$, is a step mapping with representation (3.1) on $[a, b)$. Then the following condition are equivalent:

(a) $g$ is HL integrable.

(b) The family $\left((S(\alpha)-\alpha) z_{\alpha}\right)_{\alpha \in \Lambda^{<b}}$ is summable.

If (a) or (b) holds, then $\int_{a}^{b} g(t) d t$ is the sum of the family $\left((S(\alpha)-\alpha) z_{\alpha}\right)_{\alpha \in \Lambda^{<b}}$.

Proof Assume first that the family $\left((S(\alpha)-\alpha) z_{\alpha}\right)_{\alpha \in \Lambda^{<b}}$ is summable. Denote by $\sigma(\gamma)$ the sum of $\left((S(\alpha)-\alpha) z_{\alpha}\right)_{\alpha \in \Lambda^{<}}, \gamma \in \Lambda$. We shall show that the mapping $f:[a, b) \rightarrow E$, defined by

$$
f(t)=\sigma(\gamma)+(t-\gamma) z_{\gamma}, \quad t \in[\gamma, S(\gamma)), \gamma \in \Lambda^{<b},
$$

is a CD primitive of $g$. It follows from (3.1) and (3.2) that

$$
f^{\prime}(t)=z_{\gamma}=g(t), \quad t \in(\gamma, S(\gamma)), \gamma \in \Lambda^{<b} .
$$

Thus, $f^{\prime}(t)=g(t)$ for every $t \in[a, b) \backslash \Lambda^{<b}$. In particular, $f$ is continuous in $[a, b) \backslash \Lambda^{<b}$. To prove that $f$ is continuous at every point of $[a, b)$, it suffices to prove continuity at every point $\gamma \in \Lambda^{<b}$. Since $f(t)=(t-a) z_{a}, a \leq t<S(a)$, then $f$ is right continuous at $\gamma=a$. If $\gamma \in \Lambda^{<b}$ is a successor, i.e., $\gamma=S(\beta)$ for some $\beta \in \Lambda^{<b}$, then

$$
f(t)= \begin{cases}\sigma(\beta)+(t-\beta) z_{\beta}, & t \in[\beta, \gamma)), \\ \sigma(\gamma)+(t-\gamma) z_{\gamma}, & t \in[\gamma, S(\gamma)) .\end{cases}
$$

Applying the defining condition (s) of summability, we obtain

$$
\lim _{t \rightarrow \gamma_{-}} f(t)=\sigma(\beta)+(S(\beta)-\beta) z_{\beta}=\sigma(\gamma)=\lim _{t \rightarrow \gamma_{+}} f(t) .
$$

Thus, $f$ is continuous at $\gamma=S(\beta), \beta \in \Lambda^{<b}$.

Assume next that $\gamma$ is not a successor. Given $\epsilon>0$, there exists by condition (s)(ii) of summability such a $\beta_{\epsilon} \in \Lambda^{<\gamma}$ that

$$
\|\sigma(\beta)-\sigma(\gamma)\|<\epsilon \quad \text { whenever } \beta \in \Lambda \text { and } \beta_{\epsilon} \leq \beta<\gamma .
$$

If $t \in\left(\beta_{\epsilon}, \gamma\right)$, there exists $\beta \in \Lambda, \beta_{\epsilon} \leq \beta<\gamma$, such that $t \in[\beta, S(\beta))$. Thus,

$$
\|f(t)-f(\gamma)\|=\left\|\sigma(\beta)+(t-\beta) z_{\beta}-\sigma(\gamma)\right\|<\epsilon+\left\|(t-\beta) z_{\beta}\right\| .
$$

Since also $\beta_{\epsilon} \leq S(\beta)<\gamma$, and since

$$
\left\|(t-\beta) z_{\beta}\right\| \leq\left\|(S(\beta)-\beta) z_{\beta}\right\|=\|\sigma(S(\beta))-\sigma(\beta)\|,
$$

then

$$
\|f(t)-f(\gamma)\|<\epsilon+\|\sigma(S(\beta))-\sigma(\beta)\| \leq \epsilon+\|\sigma(S(\beta))-\sigma(\gamma)\|+\|\sigma(\beta)-\sigma(\gamma)\|<3 \epsilon .
$$


This holds for every $t \in\left[\beta_{\epsilon}, \gamma\right)$. Thus, $\lim _{t \rightarrow \gamma-} f(t)=f(\gamma)$. If $t \in[\gamma, S(\gamma))$, then

$$
f(t)=\sigma(\gamma)+(t-\gamma) z_{\gamma}, \quad t \in[\gamma, S(\gamma)) .
$$

Thus, $\lim _{t \rightarrow \gamma_{-}} f(t)=\sigma(\gamma)=f(\gamma)=\lim _{t \rightarrow \gamma+} f(t)$. This proves that $f$ is continuous at $\gamma$.

The above proof shows that $f$ is continuous in $[a, b)$, and that $f^{\prime}(t)=g(t)$ in the complement of the well ordered, and hence countable subset $\Lambda^{<b}$ of $[a, b)$. Thus, $f$ is a CD primitive of $g$, so that $g$ is locally HL integrable on $[a, b)$ by Lemma 2.3. Using condition (s), it can be shown ( $c f$. the proof of Proposition 3.3) that $f(t) \rightarrow \sigma(b)$ as $t \rightarrow b-$. Thus, $\int_{a}^{t} g(s) d s=f(t)-f(a)=f(t) \rightarrow \sigma(b)$ as $t \rightarrow b-$. Thus, $g$ is HL integrable because HL integrability encloses improper integrals on finite intervals by Remarks 2.2. Hence, (b) implies (a).

Conversely, assume that the mapping $g:[a, b] \rightarrow E$ satisfies (3.1), and is HL integrable on $[a, b]$. We show by the Principle of Transfinite Induction that the family $\left((S(\alpha)-\alpha) z_{\alpha}\right)_{\alpha \in \Lambda^{<\gamma}}$ is summable for every $\gamma \in \Lambda$. Assume that $\gamma \in \Lambda$, and that $\left((S(\alpha)-\alpha) z_{\alpha}\right)_{\alpha \in \Lambda^{<\beta}}$ is summable for every $\beta \in \Lambda^{<\gamma}$. If $\gamma$ is a successor, i.e., $\gamma=S(\beta)$, then $\beta \in \Lambda^{<\gamma}$, where the sum $\sigma(\beta)$ of $\left((S(\alpha)-\alpha) z_{\alpha}\right)_{\alpha \in \Lambda^{<\beta}}$ exists in $E$. This result and the defining condition (s) of summability imply that $\left((S(\alpha)-\alpha) z_{\alpha}\right)_{\alpha \in \Lambda^{<\gamma}}$ is summable, and $\sigma(\gamma)=\sigma(\beta)+(S(\beta)-\beta) z_{\beta}$. Assume next that $\gamma$ is not a successor. Because $\left((S(\alpha)-\alpha) z_{\alpha}\right)_{\alpha \in \Lambda^{<\beta}}$ is summable for every $\beta \in \Lambda^{<\gamma}$, it follows from first part of this proof that for $\beta \in \Lambda^{<\gamma}, g$ is HL integrable on $[a, \beta]$, and that (3.2) defines continuous mapping $f$ on $[a, \beta]$. Thus,

$$
\sigma(\beta)=f(\beta)=\int_{a}^{\beta} g(s) d s, \quad \text { for every } \beta \in \Lambda^{<\gamma} .
$$

Because $g$ is HL integrable on $[a, \gamma]$, then $\lim _{\beta \rightarrow \gamma-} \int_{a}^{\beta} g(s) d s$ exists and is equal to $\int_{a}^{\gamma} g(s) d s$ by Remarks 2.2. Consequently, $\lim _{\beta \rightarrow \gamma_{-}} \sigma(\beta)$ exists, so that $\left((S(\alpha)-\alpha) z_{\alpha}\right)_{\alpha \in \Lambda^{<\gamma}}$ is summable.

The above results imply by the Principle of Transfinite Induction that the family $((S(\alpha)-$ $\left.\alpha) z_{\alpha}\right)_{\alpha \in \Lambda^{<\gamma}}$ is summable for every $\gamma \in \Lambda$. In particular, $\left((S(\alpha)-\alpha) z_{\alpha}\right)_{\alpha \in \Lambda^{<b}}$ is summable. Thus (a) implies (b).

If (a) or (b) are valid, then both of them are valid by the above proof. Thus the mapping $f$, defined by (3.2), is a primitive of $g$, whence $\int_{a}^{b} g(s) d s=f(b)-f(a)=\sigma(b)-\sigma(a)=\sigma(b)$. This proves the last conclusion.

When integrability and summability are local, we have the following result.

Proposition 3.2 Let $\Lambda$ be a well ordered subset of a real interval $[a, b),-\infty<a<b \leq \infty$, such that $\min \Lambda=a$ and $\sup \Lambda=b$. Assume that $g:[a, b) \rightarrow E$ is a step mapping defined on $[a, b)$ by (3.1). Then the following condition are equivalent:

(a) $g$ is locally $H L$ integrable.

(b) The family $\left((S(\alpha)-\alpha) z_{\alpha}\right)_{\alpha \in \Lambda^{<b}}$ is locally summable.

If (a) or (b) holds, and $c \in(a, b)$, then $\int_{a}^{c} g(t) d t=f(c)$, where $f:[a, b) \rightarrow \mathbb{R}$ is defined on $[a, b)$ by (3.2).

Proof Assume first that the family $\left((S(\alpha)-\alpha) z_{\alpha}\right)_{\alpha \in \Lambda^{<b}}$ is locally summable. Because $\Lambda=$ $\Lambda^{<b}$, then (3.2) defines a mapping $f:[a, b) \rightarrow \mathbb{R}$, and $f^{\prime}(t)=g(t)$ for each $t \in[a, b) \backslash \Lambda$. As 
in the proof of Proposition 3.1 it can be shown that $f$ is continuous. Thus, by Lemma 2.3, $g$ is locally HL integrable, so that (b) implies (a).

Conversely, assume that the mapping $g:[a, b) \rightarrow E$, defined by (3.1), is locally HL integrable on $[a, b)$. As in the proof of Proposition 3.1 it can be shown that the family $\left((S(\alpha)-\alpha) z_{\alpha}\right)_{\alpha \in \Lambda<\gamma}$ is summable for every $\gamma \in \Lambda$, so that $\left((S(\alpha)-\alpha) z_{\alpha}\right)_{\alpha \in \Lambda}$ is locally summable. Thus, (a) implies (b).

If (a) or (b) holds, then they both are valid. Assume that $c \in(a, b)$. Because the mapping $f$, defined by (3.2), is a CD primitive of $g$, it follows from the last conclusion of Lemma 2.3 that $\int_{a}^{c} g(s) d s=f(c)-f(a)=f(c)$.

As an application of Lemma 2.4 and Propositions 3.1 and 3.2, we obtain the following result.

Proposition 3.3 Assume that $g:[a, \infty] \rightarrow E$ is a step mapping satisfying (3.1) with $b=\infty$. Then $g$ is HK integrable if and only if the family $\left((S(\alpha)-\alpha) z_{\alpha}\right)_{\alpha \in \Lambda^{<\infty}}$ is summable.

Proof Assume first that the family $\left((S(\alpha)-\alpha) z_{\alpha}\right)_{\alpha \in \Lambda^{<\infty}}$ is summable. Then it is also locally summable, where $g$ is locally HL integrable by Proposition 3.3. Thus, $g$ is also locally HK integrable. Denote by $\sigma(\gamma)$ the sum of $\left((S(\alpha)-\alpha) z_{\alpha}\right)_{\alpha \in \Lambda^{<\gamma}}, \gamma \in \Lambda$. Let $f:[a, \infty) \rightarrow E$ be defined by

$$
f(t)=\sigma(\gamma)+(t-\gamma) z_{\gamma}, \quad t \in[\gamma, S(\gamma)), \gamma \in \Lambda^{<\infty} .
$$

Because the family $\left((S(\alpha)-\alpha) z_{\alpha}\right)_{\alpha \in \Lambda^{<\infty}}$ is summable, then $\infty$ is a limit member of $\Lambda$. Given $\epsilon>0$, there exists by condition (s)(ii) of summability such a $\beta_{\epsilon} \in \Lambda^{<\infty}$ that

$$
\|\sigma(\beta)-\sigma(\infty)\|<\epsilon \quad \text { whenever } \beta \in \Lambda \text { and } \beta_{\epsilon} \leq \beta<\infty .
$$

If $t \in\left(\beta_{\epsilon}, \infty\right)$, there exists $\beta \in \Lambda, \beta_{\epsilon} \leq \beta<\infty$, such that $t \in[\beta, S(\beta))$. Thus,

$$
\|f(t)-\sigma(\infty)\|=\left\|\sigma(\beta)+(t-\beta) z_{\beta}-\sigma(\infty)\right\|<\epsilon+\left\|(t-\beta) z_{\beta}\right\| .
$$

Since also $\beta_{\epsilon} \leq S(\beta)<\infty$, and since

$$
\left\|(t-\beta) z_{\beta}\right\| \leq\left\|(S(\beta)-\beta) z_{\beta}\right\|=\|\sigma(S(\beta))-\sigma(\beta)\|,
$$

then

$$
\|f(t)-\sigma(\infty)\|<\epsilon+\|\sigma(S(\beta))-\sigma(\beta)\| \leq \epsilon+\|\sigma(S(\beta))-\sigma(\infty)\|+\|\sigma(\beta)-\sigma(\infty)\|<3 \epsilon .
$$

This holds for every $t \in\left[\beta_{\epsilon}, \infty\right)$. Thus, $\lim _{t \rightarrow \infty} f(t)=\sigma(\infty)$. Because $\int_{a}^{t} g(s) d s=f(t)$ for each $t \in[a, \infty)$, by Proposition 3.3, then the $\operatorname{limit}_{\lim } \rightarrow \infty \int_{a}^{t} g(s) d s$ exists. This implies by Lemma 2.4 that $g$ is HK integrable on $[a, \infty]$.

Assume next that the family $\left((S(\alpha)-\alpha) z_{\alpha}\right)_{\alpha \in \Lambda^{<\infty}}$ is not summable. Then there is by Lemma 2.1 the greatest element $c$ in $\Lambda^{<\infty} \backslash\{\min \Lambda\}$ such that the family $\left((S(\alpha)-\alpha) z_{\alpha}\right)_{\alpha \in \Lambda^{<\gamma}}$ is summable for each $\gamma \in \Lambda^{<c}$. Moreover, $c$ is not a successor. In particular, the limit $\lim _{\gamma \rightarrow c} \sigma(\gamma)$ does not exist. Thus, $g$ is locally HL and HK integrable on $[a, c)$, but 
$\lim _{\gamma \rightarrow c} \int_{a}^{\gamma} g(s) d s$ does not exist. Consequently, the $\operatorname{limit}_{\lim } \rightarrow \rightarrow \int_{a}^{t} g(s) d s$ does not exist, whence Lemma 2.4 implies that $g$ is not HK integrable on $[a, c]$. Therefore, $g$ is not HK integrable on $[a, \infty]$.

Proposition 3.1 is applied in the proof of the following results.

\section{Proposition 3.4}

(a) Let $g:[a, b] \rightarrow E,-\infty<a<b<\infty$, be a step mapping that satisfies (3.1). Then $g$ is Bochner integrable if and only if the family $\left((S(\alpha)-\alpha) z_{\alpha}\right)_{\alpha \in \Lambda^{<b}}$ is absolutely summable.

(b) Let $\Lambda$ be a well-ordered subset of a real interval $[a, b),-\infty<a<b \leq \infty$, such that $\min \Lambda=a$ and $\sup \Lambda=b$. Assume that $g:[a, b) \rightarrow E$ is a step mapping defined on $[a, b)$ by (3.1). Then $g$ is locally Bochner integrable if and only if the family $\left((S(\alpha)-\alpha) z_{\alpha}\right)_{\alpha \in \Lambda^{<b}}$ is locally absolutely summable.

Proof (a) Because $g$ is by (3.1) strongly measurable, then $g$ is Bochner integrable if and only if the function $h=t \mapsto\|g(t)\|$ is Lebesgue integrable. Replacing $z_{\alpha}$ by $\left\|z_{\alpha}\right\|$ in (3.1) and in (3.2), it follows from Proposition 3.1 that $h$ is HL integrable if and only if the family $\left((S(\alpha)-\alpha)\left\|z_{\alpha}\right\|\right)_{\alpha \in \Lambda^{<b}}$ is summable. Because a real-valued function is HL integrable if and only if it is HK integrable, and nonnegative-valued function is HK integrable if and only if it is Lebesgue integrable, then $h$ is Lebesgue integrable, or equivalently $g$ is Bochner integrable, if and only if the family $\left((S(\alpha)-\alpha)\left\|z_{\alpha}\right\|\right)_{\alpha \in \Lambda^{<b}}$ is summable, or equivalently, the family $\left((S(\alpha)-\alpha) z_{\alpha}\right)_{\alpha \in \Lambda^{<b}}$ is absolutely summable.

The conclusion (b) follows from (a) and from the definitions of local integrability and local summability.

Proposition 3.5 Assume that $\Lambda$ is a well-ordered subset of a real interval $[a, b)$ such that $\min \Lambda=a$ and $\sup \Lambda=b$. Given a family $\left(z_{\alpha}\right)_{\alpha \in \Lambda}$ of $E$, let $g:[a, b] \rightarrow$ E satisfy (3.1).

(a) If $b<\infty$, then $g$ is Riemann integrable on $[a, b]$ if and only if the family $\left(z_{\alpha}\right)_{\alpha \in \Lambda}$ is bounded.

(b) If $b=\infty$, the improper Riemann integral of $g$ over $[a, b]$ exists if and only if the family $\left(z_{\alpha}\right)_{\alpha \in \Lambda^{<\gamma}}$ is bounded for every $\gamma \in \Lambda$, and the family $\left((S(\alpha)-\alpha) z_{\alpha}\right)_{\alpha \in \Lambda^{<b}}$ is summable.

Proof (a) Assume first that the family $\left(z_{\alpha}\right)_{\alpha \in \Lambda}$ is bounded. It follows from (3.1) that $g$ is bounded, and that, the set of its discontinuity points is a subset of $\Lambda$, and hence a null set. Thus, $g$ is Riemann integrable by Lemma 2.2. Conversely, if $g$ is Riemann integrable, it is bounded by Lemma 2.2. Since $g(\alpha)=z_{\alpha}$ for each $\alpha \in \Lambda^{<b}$, then the family $\left(z_{\alpha}\right), \alpha \in \Lambda$ is bounded. This proves the assertion.

(b) Assume that $b=\infty$, and that the set $\left(z_{\alpha}\right)_{\alpha \in \Lambda^{<\gamma}}$ is bounded for every $\gamma \in \Lambda$. It then follows from (3.1) that $g$ is bounded, and hence Riemann integrable on each interval $[a, c]$, $a<c<\infty$. Proposition 3.3 implies that $g$ is HK integrable on $[a, \infty]$ if the family $((S(\alpha)-$ $\left.\alpha) z_{\alpha}\right)_{\alpha \in \Lambda^{<b}}$ is summable. In this case $\lim _{c \rightarrow \infty} \int_{a}^{c} g(s) d s$ exists by Lemma 2.4. This limit is the improper Riemann integral because every integral $\int_{a}^{c} g(s) d s, a<c<\infty$, is Riemann integral. If the family $\left((S(\alpha)-\alpha) z_{\alpha}\right)_{\alpha \in \Lambda^{<b}}$ is not summable, then $g$ is not HK integrable on $[a, \infty]$, whence the improper Riemann integral over $[a, b]$ does not exist. 
Example 3.1 Let $\left(y_{n}\right)_{n=0}^{\infty}$ be a sequence in a Banach space $E$, and let $g:[0, \infty] \rightarrow E$ be defined by

$$
g(t)=y_{n}, \quad t \in[n, n+1), n \in \mathbb{N}_{0}, \quad g(\infty)=0 .
$$

Show that

(a) $g$ is HK integrable if and only if the series $\sum_{n=0}^{\infty} y_{n}$ is summable;

(b) $g$ is Bochner integrable if and only if the series $\sum_{n=1}^{\infty} y_{n}$ is absolutely summable;

(c) The improper Riemann integral of $g$ exist if and only if the series $\sum_{n=0}^{\infty} y_{n}$ is summable.

Solution Denoting $\alpha_{n}:=n, n \in \mathbb{N}_{0}, \Lambda=\mathbb{N}_{0}$ and $z_{\alpha_{n}}=y_{n}, n \in \mathbb{N}_{0}$, then $g$ can be rewritten as

$$
g(t)=z_{\alpha_{n}}, \quad t \in\left[\alpha_{n}, S\left(\alpha_{n}\right)\right), n \in \mathbb{N}_{0}, \quad g(\infty)=0 .
$$

The series $\sum_{n=0}^{\infty} y_{n}$ is summable in ordinary or absolute sense if and only if the series $\sum_{n=0}^{\infty}\left(S\left(\alpha_{n}\right)-\alpha_{n}\right) z_{\alpha_{n}}$ has the same property. Moreover, if $\sum_{n=0}^{\infty} y_{n}$ is summable, then the set $\left\{z_{\alpha_{n}} \mid n \in \mathbb{N}_{0}\right\}$ is bounded. Thus, (a) follows from Proposition 3.3, (b) from Proposition 3.4, and (c) from Proposition 3.5.

Example 3.2 Let $\left(y_{n}\right)_{n=0}^{\infty}$ be a sequence in a Banach space $E$, and let $g:[0,1] \rightarrow E$ be defined by

$$
g(t)=y_{n}, \quad t \in\left[1-2^{-n+1}, 1-2^{-n}\right), n \in \mathbb{N}_{0}, \quad g(1)=0 .
$$

Show that

(a) properties: $g$ is HL integrable, and the series $\sum_{n=1}^{\infty} 2^{-n} y_{n}$ is summable, are equivalent;

(b) $g$ is Bochner integrable if and only if the series $\sum_{n=1}^{\infty} 2^{-n} y_{n}$ is absolutely summable;

(c) The improper Riemann integral of $g$ exists if and only if the sequence $\left(y_{n}\right)$ is bounded.

Solution The correspondence $n \leftrightarrow \alpha_{n}:=1-2^{-n+1}$ is an order preserving isomorphism between $\mathbb{N}$ and $\Lambda=\left\{\alpha_{n} \mid n \in \mathbb{N}_{0}\right\}$. Denoting $z_{\alpha_{n}}=y_{n}, n \in \mathbb{N}$, and noticing that $S\left(\alpha_{n}\right)-\alpha_{n}=$ $1-2^{-n}-\left(1-2^{-n+1}\right)=2^{-n}$, then $g$ can be rewritten as

$$
g(t)=z_{\alpha_{n}}, \quad t \in\left[\alpha_{n}, S\left(\alpha_{n}\right)\right), n \in \mathbb{N}_{0}, \quad g(1)=0 .
$$

The series $\sum_{n=1}^{\infty} 2^{-n} y_{n}$ is summable in ordinary or absolute sense if and only if the series $\sum_{n=1}^{\infty}\left(S\left(\alpha_{n}\right)-\alpha_{n}\right) z_{\alpha_{n}}$ has the same property. Thus, the conclusions of (a), (b) and (c) follow from Propositions 3.1, 3.4 and 3.5, respectively.

In view of Example 2.1, the preceding example can be generalized as follows.

Example 3.3 Given $m \in \mathbb{N}$, let $\Lambda_{m}=\left\{\alpha\left(n_{0}, \ldots, n_{m}\right) \mid n_{0}, \ldots, n_{m} \in \mathbb{N}_{0}\right\}$ be defined by (2.2). Then $S\left(\alpha\left(n_{0}, \ldots, n_{m}\right)\right)=\alpha\left(n_{0}, \ldots, n_{m}+1\right), m, n_{0}, \ldots, n_{m} \in \mathbb{N}_{0}$ so that

$$
S\left(\alpha\left(n_{0}, \ldots, n_{m}\right)\right)-\alpha\left(n_{0}, \ldots, n_{m}\right)=2^{-\sum_{k=0}^{m} n_{k}-m-1} .
$$


Thus, if $\left(z_{\alpha\left(n_{0}, \ldots, n_{m}\right)}\right)_{\alpha\left(n_{0}, \ldots, n_{m}\right) \in \Lambda_{m}}$ is such a family of real numbers that the family

$$
\left(2^{-\sum_{k=0}^{m} n_{k}-m-1} z_{\alpha\left(n_{0}, \ldots, n_{m}\right)}\right)_{\alpha\left(n_{0}, \ldots, n_{m}\right) \in \Lambda_{m}}
$$

is summable, then the mapping $g:[0,1] \rightarrow E$, defined by

$$
\begin{aligned}
& g(t)=z_{\alpha\left(n_{0}, \ldots, n_{m}\right)}, \quad t \in\left[\alpha\left(n_{0}, \ldots, n_{m}\right), S\left(\alpha\left(n_{0}, \ldots, n_{m}\right)\right)\right), \alpha\left(n_{0}, \ldots, n_{m}\right) \in \Lambda_{m}, \\
& g(1)=0,
\end{aligned}
$$

is HL integrable by Proposition 3.1. According to Proposition 3.4, $g$ is Bochner integrable if and only if the above family is absolutely summable. If the family $\left(z_{\alpha\left(n_{0}, \ldots, n_{m}\right)}\right)_{\alpha\left(n_{0}, \ldots, n_{m}\right) \in \Lambda_{m}}$, is bounded, then $g$ is Riemann integrable by Proposition 3.5.

Remarks 3.1 Example 3.1 contains the results of [7, Theorem 4(a) and (c)]. As for related results, see [12].

Let $g:[0,1) \rightarrow E$ be as in Example 3.2, and let $h:[0,1] \rightarrow E$ be defined by

$$
h(t)=y_{n}, \quad t \in\left(2^{-n}, 2^{-n+1}\right], n \in \mathbb{N}_{0}, \quad h(0)=0 .
$$

Because $h(t)=g(1-t), t \in(0,1)$, it follows from Example 3.2 that

(a) properties: $h$ is HL integrable, and the series $\sum_{n=1}^{\infty} 2^{-n} y_{n}$ is summable, are equivalent, and that

(b) $h$ is Bochner integrable if and only if the series $\sum_{n=1}^{\infty} 2^{-n} y_{n}$ is absolutely summable.

The result (a) contains the result (a) of [6, Proposition 5.4.1] and improves the results of [6, Proposition 5.4.2] and [5, Example], where unconditional convergence of series $\sum_{n=1}^{\infty} 2^{-n} y_{n}$ is shown to imply the HL integrability of $h$. The result (b) is equivalent to the result (c) of [6, Proposition 5.4.1].

Example 3.3 can be used to generalize further the results of $[5,6]$ cited above.

In [13], a notion of convergence for multiple series is defined and shown to be equivalent to the HK integrability of the associated step function over an unbounded multidimensional interval.

\section{On HK, HL, Bochner and Riemann integrability of right regulated mappings}

Applying Lemmas 2.5 and 2.6 and the results derived for step mappings in Section 3, we shall derive in this section criteria for HK, HL, Bochner and Riemann integrability of right regulated mappings.

Proposition 4.1 Given a right regulated mapping $g:[a, b] \rightarrow E,-\infty<a<b<\infty$, and a positive number $\epsilon$, let $\Lambda_{\epsilon}$ be the well ordered subset of $[a, b]$ defined by (2.6). Then the following properties are equivalent.

(a) $g$ is HL integrable.

(b) The step mapping $g_{\epsilon}:[a, b] \rightarrow E$, defined by

$$
g_{\epsilon}(t)=g(\beta+), \quad t \in[\beta, S(\beta)), \beta \in \Lambda_{\epsilon}^{<b}, \quad g_{\epsilon}(b)=g(b),
$$

is HL integrable.

(c) The family $((S(\beta)-\beta) g(\beta+))_{\Lambda_{\epsilon}^{c b}}$ is summable. 
Proof It follows from Lemma 2.5 and (4.1) that $\left\|g_{\epsilon}(t)-g(t)\right\| \leq \epsilon$ whenever $t \in(\beta, S(\beta))$ and $\beta \in \Lambda_{\epsilon}^{<b}$. Because $g$ is strongly measurable by Lemma 2.6 and $g_{\epsilon}$ is strongly measurable by definition (4.1), then $g_{\epsilon}-g$ is Bochner integrable, and hence also HL integrable. Consequently, if $g$ is HL integrable, then $g_{\epsilon}=g+\left(g_{\epsilon}-g\right)$ is HL integrable, and if $g_{\epsilon}$ is HL integrable, then $g=g_{\epsilon}-\left(g-g_{\epsilon}\right)$ is HL integrable. This proves that (a) and (b) are equivalent. The equivalence of (b) and (c) follows from Proposition 3.1.

Proposition 3.4 and the proof of Proposition 4.1 is used to prove the following results.

Proposition 4.2 Let $g:[a, b] \rightarrow E,-\infty<a<b<\infty$, be right regulated, and let $\epsilon$ be a positive number. Let $\Lambda_{\epsilon}$ be the well-ordered subset of $[a, b]$ defined by (2.6). Then the following properties are equivalent.

(a) $g$ is Bochner integrable.

(b) The mapping $g_{\epsilon}:[a, b] \rightarrow E$, defined by (4.1), is Bochner integrable.

(c) The family $((S(\beta)-\beta) g(\beta+))_{\Lambda_{\epsilon}^{<b}}$ is absolutely summable.

Proof It follows from the proof of Proposition 4.1 that $g_{\epsilon}-g$ is Bochner integrable. Hence, if $g$ is Bochner integrable, then $g_{\epsilon}=g+\left(g_{\epsilon}-g\right)$ is Bochner integrable, and if $g_{\epsilon}$ is Bochner integrable, then $g=g_{\epsilon}-\left(g-g_{\epsilon}\right)$ is Bochner integrable. This implies that (a) and (b) are equivalent. The equivalence of (b) and (c) follows from Proposition 3.4.

Proposition 4.3 Let $g:[a, b] \rightarrow E,-\infty<a<b<\infty$, be right regulated, and let $\Lambda_{\epsilon}, \epsilon>0$, be the well ordered subset of $[a, b]$ defined by (2.6). Then the following properties are equivalent.

(a) $g$ is Riemann integrable.

(b) $g$ is bounded.

(c) The mapping $h_{\epsilon}:[a, b] \rightarrow E$, defined by

$$
h_{\epsilon}(t)=g(\beta+), \quad t \in[\beta, S(\beta)), \beta \in \Lambda_{\epsilon}^{<b}, \quad h_{\epsilon}(\beta)=g(\beta), \quad \beta \in \Lambda_{\epsilon},
$$

is HL integrable is Riemann integrable.

(d) The families $(g(\beta))_{\beta \in \Lambda_{\epsilon}^{<b}}$ and $(g(\beta+))_{\beta \in \Lambda_{\epsilon}^{<b}}$ are bounded.

Proof The set of discontinuity points of $g$ is countable, whence the equivalence of (a) and (b) follows [9, Theorem 24.45]. The mapping $h_{\epsilon}-g$ is bounded and has only a countable number of discontinuities, so that it is Riemann integrable. Hence, if $g$ is Riemann integrable, then $h_{\epsilon}=g+\left(h_{\epsilon}-g\right)$ is Riemann integrable, and if $h_{\epsilon}$ is Riemann integrable, then $g=h_{\epsilon}-\left(g-h_{\epsilon}\right)$ is Riemann integrable. This implies that (a) and (c) are equivalent. The proof of the equivalence of (c) and (d) is similar to that of Proposition 3.5.

Now we are in position to prove the results presented in the Introduction.

Theorem 4.1 (the fundamental theorem of calculus for right regulated mappings) Assume that a mapping $g: I \rightarrow E,-\infty<\min I<\sup I \leq \infty$ is right regulated.

(a) $g$ is locally HL integrable if and only if it has a CD primitive.

(b) $g$ is locally Bochner integrable if and only if it has a locally absolutely continuous $C D$ primitive. 
(c) $g$ is locally Riemann integrable if and only if it is locally bounded, in which case $g$ has a locally Lipschitz continuous CD primitive.

Proof (a) Denote $a=\min I$ and $c=\sup I$. It follows from Lemma 2.3 that if $g$ has a CD primitive, then $g$ is locally HL integrable. To prove converse, assume that $g$ is locally HL integrable. Given $b \in(a, c)$, define for each $n \in \mathbb{N}$ the step mapping $g_{n}:[a, b] \rightarrow E$ by

$$
g_{n}(t)=g(\beta+), \quad t \in[\beta, S(\beta)), \beta \in \Lambda_{\frac{1}{n}}^{<b}, \quad g_{n}(b)=g(b) .
$$

Because $g$ is HL integrable on $[a, b]$, it follows from Proposition 4.1 that the family $((S(\beta)-$ $\left.\beta) g_{n}(\beta+)\right)_{\Lambda_{\frac{1}{n}}^{<b}}$ is summable for every $n \in \mathbb{N}$. Denote by $\sigma_{n}(\gamma)$ the sum of the family $((S(\beta)-$ $\beta) g(\beta+))_{\Lambda_{\frac{1}{n}}^{<\gamma}}, \gamma \in \Lambda_{\frac{1}{n}}^{<b}$. The proof of Proposition 3.1 that implies that for each $n \in \mathbb{N}$ the mapping $f_{n}^{n}:[a, b) \rightarrow E$, defined by

$$
f_{n}(t)=\sigma_{n}(\gamma)+(t-\gamma) g(\gamma+), \quad t \in[\gamma, S(\gamma)), \gamma \in \Lambda_{\frac{1}{n}}^{<b},
$$

is a CD primitive of $g_{n}$. Thus, for each $n \in \mathbb{N}$, the mapping $f_{n}$ is continuous, $f_{n}^{\prime}(t)=g_{n}(t)$ for all $t \in[a, b] \backslash \Lambda_{\frac{1}{n}}$, and $f_{n}(a)=\sigma_{n}(a)=0$. Moreover, if $g(\beta+)=g(\beta)$ for $\beta \in Z=\bigcup_{n=1}^{\infty} \Lambda_{n}$, then $\left\|g_{n}(t)-g(t)\right\| \leq \frac{1}{n}$ for each $t \in[a, b)$ by Lemma 2.5 and (4.3), so that the sequence $\left(g_{n}\right)_{n=1}^{\infty}$ converges uniformly to $g$. Consequently, it follows from $[1,(8.6 .4)]$ that the sequence $\left(f_{n}\right)_{n=1}^{\infty}$ converges uniformly on $[a, b)$ to a continuous mapping $f:[a, b) \rightarrow E$, and $f^{\prime}(t)=g(t)$ for each $t \in[a, b) \backslash Z$. $f$ has these properties also when right continuity of $g$ in $Z$ is not assumed. Because $Z$ is countable, then $f$ is a CD primitive of the restriction of $g$ to $[a, b]$.

Choose an increasing sequence $\left(c_{n}\right)_{n=1}^{\infty}$ from $(a, c)$ so that it converges to $c$. The interval $[a, c)$ is the union of increasing sequence of compact intervals $\left[a, c_{n}\right]$, and $g$ is HL integrable on these compact intervals. By the above proof the restriction of $g$ to $\left[a, c_{n}\right]$ has a CD primitive $f_{n}$, and $f_{n}(a)=0$ for each $n \in \mathbb{N}$. Defining

$$
f(t)=\left\{\begin{array}{l}
f_{1}(t)-f(a), \quad t \in\left[a, c_{1}\right), \\
f_{n+1}(t)-f_{n+1}(a), \quad t \in\left[c_{n}, c_{n+1}\right), n \in \mathbb{N},
\end{array}\right.
$$

we obtain a mapping $f:[a, c) \rightarrow E$ which is a CD primitive of $g$ (cf. Remark after [1, (8.7.1)]).

(b) If $g$ has a locally absolutely continuous CD primitive $f$, then $g$ is locally Bochner integrable by [2, Theorem 1.4.6]. Conversely, assume that $g$ is locally Bochner integrable, and let $[a, b]$ be a compact subinterval of $I$. Then $g$ is Bochner integrable on $[a, b]$, whence the restriction of $g$ to $[a, b]$ has by [2, Theorem 1.4.6] an absolutely continuous primitive $h:[a, b] \rightarrow E . g$ is also locally HL integrable by [6, Proposition 3.6.3 and Theorem 5.1.4]. Thus, $g$ has by the proof of (a) a CD primitive $f: I \backslash\{\sup I\} \rightarrow E$. It follows from Lemma 2.3 and from the definition (2.4) of the Henstock-Kurzweil integral that $f(x)-f(a)=h(x)-$ $h(a)$, i.e., $f(x)=h(x)+f(a)-h(a)$ for all $x \in[a, b]$. Thus, $f$ is absolutely continuous on $[a, b]$. Consequently, $f$ is an absolutely continuous CD primitive of the restriction of $g$ to $[a, b]$.

(c) Assume that $g$ is locally Riemann integrable, and let $[a, b]$ be a compact subinterval of $I$. It follows from Proposition 4.3 that $g$ is Riemann integrable on $[a, b]$ if and only if $g$ is bounded on $[a, b]$, in which case there is such a positive constant $M$ that $\|g(t)\| \leq M$ for each $t \in[a, b]$. Because $g$ is also locally HL integrable, it has a CD primitive $f$ by the 
proof of (a), and $f(b)-f(a)=\int_{a}^{b} g(t) d t$ by Lemma 2.3. Thus, $\|f(b)-f(a)\| \leq \int_{a}^{b}\|g(t) d t\| \leq$ $M(b-a)$. This holds for every compact subinterval $[a, b]$ of $I$, whence $f$ is locally Lipschitz continuous.

The following results are easy consequences of the results of Theorem 4.1 and Lemma 2.4 and the definitions of integrals and improper integrals.

Corollary 4.1 Let $g: I \rightarrow E,-\infty<\min I<\sup I \leq \infty$ be right regulated.

(a) $g$ is HL integrable when I is bounded if and only if $g$ has a CD primitive that has the left limit at $\sup I$.

(b) $g$ is HK integrable if it has a CD primitive that has the left limit at $\sup I$.

(c) $g$ is Bochner integrable if and only if the function it has a locally absolutely continuous $C D$ primitive that has the left limit at $\sup I$.

(d) $g$ is Riemann integrable if and only if it is bounded and I is bounded.

(e) The improper Riemann integral of $g$ from $\min I$ to $\sup I$ if $g$ is locally bounded, and its $C D$ primitive has the left limit at $\sup I$.

The next result follows from Lemma 2.1 and Propositions 4.1, 4.2 and 4.3.

Theorem 4.2 Let $g: I \rightarrow E,-\infty<\min I<\sup I \leq \infty$ be right regulated.

(a) For each compact subinterval $[a, b]$ of $I$, either $g$ is Riemann integrable on $[a, b]$, or there exists the greatest number $c_{1}$ in $(a, b]$ such that $g$ is locally Riemann integrable on $\left[a, c_{1}\right)$.

(b) For each compact subinterval $[a, b]$ of $I$, either $g$ is Bochner integrable on $[a, b]$, or there exists the greatest number $c_{2}$ in $(a, b]$ such that $g$ is locally Bochner integrable on $\left[a, c_{2}\right)$.

(c) For each compact subinterval $[a, b]$ of I, either $g$ is HL integrable on $[a, b]$, or there exists the greatest number $c_{3}$ in $(a, b]$ such that $g$ is locally HL integrable on $\left[a, c_{3}\right)$.

Proof Let $[a, b]$ be a compact subinterval of $I$, let $\epsilon$ be a positive number, and let $\Lambda_{\epsilon}$ be the well ordered subset of $[a, b]$ defined by (2.6).

(a) According to Lemma 2.1(a) the family $(g(\beta+))_{\beta \in \Lambda_{\epsilon} b}$ is bounded, or there exists the greatest number $c_{1}$ in $\Lambda_{\epsilon}^{<b}, c_{1}>a$, such that the family $(g(\beta+))_{\beta \in \Lambda_{\epsilon}^{<\gamma}}$ is bounded for every $\gamma \in \Lambda_{\epsilon}^{<c_{1}}$. This result and Proposition 4.3 imply that $g$ is Riemann integrable either on $[a, b]$, or on $[a, \gamma]$, for every $\gamma \in \Lambda_{\epsilon}^{<c_{1}}$. This proves (a) because $c_{1}$ is by Lemma 2.1(d) not a successor.

(b) By Lemma 2.1(b) the family $((S(\beta)-\beta) g(\beta+))_{\beta \in \Lambda_{\epsilon}^{<b}}$ is absolutely summable, or there exists the greatest number $c_{2}$ in $\Lambda_{\epsilon}^{<b}, c_{1}>a$, such that the family $((S(\beta)-\beta) g(\beta+))_{\beta \in \Lambda_{\epsilon}^{<\gamma}}$ is absolutely summable for every $\gamma \in \Lambda_{\epsilon}^{<c_{2}}$. This result implies by Proposition 4.2 that $g$ is Bochner integrable either on $[a, b]$, or on $[a, \gamma]$, for every $\gamma \in \Lambda_{\epsilon}^{<c_{2}}$. This implies conclusion (b), since by Lemma 2.1 (d) $c_{2}$ is not a successor.

(c) The proof of (c) is similar to that of (b) when absolute summability is replaced by summability, Lemma 2.1(b) by Lemma 2.1(c), and Proposition 4.2 by Proposition 4.1.

Example 4.1 Denote $c_{0}=\left\{x=\left(x_{i}\right)_{i=1}^{\infty} \mid x_{i} \in \mathbb{R}, i \in \mathbb{N}, \lim _{i \rightarrow \infty} x_{i}=0\right\} . c_{0}$ is a vector space with respect to componentwise addition and scalar multiplication, and $\|x\|=\sup _{i \in \mathbb{N}}\left|x_{i}\right|$ defines 
a Banach norm in $c_{0}$. Define a mapping $g_{0}: \mathbb{R}_{+} \rightarrow c_{0}$ by

$$
\begin{aligned}
g_{0}(t)= & \left(\sum _ { n = 1 } ^ { \infty } \frac { 1 } { n ^ { 2 } i } \left(2(n t-\lfloor n t\rfloor) \cos \left(\frac{\pi}{2(n t-\lfloor n t\rfloor)}\right)\right.\right. \\
& \left.\left.+\frac{\pi}{2} \sin \left(\frac{\pi}{2(n t-\lfloor n t\rfloor)}\right)\right)\right)_{i=1}^{\infty}, \quad t \in \mathbb{R}_{+},
\end{aligned}
$$

where $\lfloor n t\rfloor=m, m-1<n t \leq m, m=0,1, \ldots, g_{0}$ is right regulated. The set $\mathbb{Q}_{+}$of all rational numbers of $\mathbb{R}_{+}$is the set of discontinuity points $g_{0}(c f .[14,(236)])$. Moreover, all these discontinuities are of second kind. A CD primitive of $g$ is given by

$$
f(t)=\left(\sum_{n=1}^{\infty} \frac{(n t-\lfloor n t\rfloor)^{2}}{n^{3} i} \cos \left(\frac{\pi}{2(n t-\lfloor n t\rfloor)}\right)\right)_{i=1}^{\infty}, \quad t \in \mathbb{R}_{+}
$$

Because $g$ is bounded, it is also locally Riemann integrable by Theorem 4.1.

The mapping $g=t \mapsto e^{-t} g_{0}(t)$ has the improper Riemann integral $\int_{0}^{\infty} g(t) d t$.

Example 4.2 Let $g_{0}$ and $f_{0}$ be defined by (4.5) and (4.6). Define mappings $g_{m}: \mathbb{R}_{+} \rightarrow c_{0}$, $m \in \mathbb{N}$, by

$$
g_{m}(t)=g_{0}(t)+\left(\frac{1}{i} \sum_{n=1}^{i \wedge m}\left(\cos \left(\frac{\pi}{2(n t-\lfloor n t\rfloor)}\right)+\frac{\pi \sin \left(\frac{\pi}{2(n t-\lfloor n t\rfloor)}\right)}{2(n t-\lfloor n t\rfloor)}\right)\right)_{i=1}^{\infty}, \quad t \in \mathbb{R}_{+},
$$

where $i \wedge m=\min \{i, m\} . g_{m}$ is right regulated, and $\mathbb{Q}_{+}$is the set of discontinuity points, of second kind, of $g_{m}$. for all $m \in \mathbb{N}$. The mapping $f_{m}: \mathbb{R}_{+} \rightarrow c_{0}$, defined by

$$
f_{m}(t)=f_{0}(t)+\left(\sum_{n=i}^{i \wedge m} \frac{(n t-\lfloor n t\rfloor)^{2}}{n i} \cos \left(\frac{\pi}{2(n t-\lfloor n t\rfloor)}\right)\right)_{i=1}^{\infty}, \quad t \in \mathbb{R}_{+}
$$

is a $C D$ primitive of $g_{m}$ for each $m \in \mathbb{N}$. It then follows from Theorem 4.1 that the mappings $g_{m}$ are locally HL integrable. On the other hand, $g_{m}$ is neither locally Bochner integrable nor locally Riemann integrable for any $m \in \mathbb{N}$.

The mappings $t \mapsto e^{-t} g_{m}(t)$ are HK integrable on $\mathbb{R}_{+}$.

Example 4.3 Let $g_{0}$ and $f_{0}$ be defined by (4.5) and (4.6). Define mappings $g^{m}: \mathbb{R}_{+} \rightarrow c_{0}$, $m \in \mathbb{N}$, by

$$
g^{m}(t)=g_{0}(t)+\left(\frac{1}{i} \sum_{n=1}^{i \wedge m} \frac{1}{2 \sqrt{[n t]-n t}}\right)_{i=1}^{\infty}, \quad t \in \mathbb{R}_{+} .
$$

$g^{m}$ is right regulated, and $\mathbb{Q}_{+}$is its set of discontinuity points, of second kind, for every $m \in \mathbb{N}$. The mappings $f^{m}: \mathbb{R}_{+} \rightarrow c_{0}$, defined by

$$
f^{m}(t)=f_{0}(t)+\left(\sum_{n=1}^{i \wedge m} \frac{\lfloor n t\rfloor-\sqrt{\lfloor n t\rfloor-n t}}{n i}\right)_{i=1}^{\infty}, \quad t \in \mathbb{R}_{+}
$$


are absolutely continuous, and $\left(f^{m}\right)^{\prime}(t)=g^{m}(t)$ for all $t \in \mathbb{R}_{+} \backslash \mathbb{Q}_{+}$. Hence, every $g^{m}$ is locally Bochner integrable by Theorem 4.1. But $g^{m}$ is not locally bounded, and hence not locally Riemann integrable, for any $m \in \mathbb{N}$.

The mappings $t \mapsto e^{-t} g^{m}(t)$ are Bochner integrable on $\mathbb{R}_{+}$.

Remarks 4.1 Integrability results derived in Propositions 4.1, 4.2 and 4.3, and in Theorems 4.1 and 4.2 for right regulated mappings have also analogous counterparts for left regulated mappings.

\section{Applications to impulsive differential equations}

Let $E$ be a Banach space and $[a, c),-\infty<a<c \leq \infty$, a real interval. Denote by $H L_{\text {loc }}([a, c), E)$ the space of all locally $\mathrm{HL}$ integrable mappings from $[a, c)$ to $E$. Almost everywhere (a.e.) equal mappings of $H L_{\mathrm{loc}}([a, c), E)$ are identified. Consider the following impulsive problem:

$$
u^{\prime}(t)=f(t, u) \quad \text { a.e. on }[a, c), \quad \Delta u(\lambda)=D(\lambda, u), \quad \lambda \in \Lambda,
$$

where $f:[a, b) \times H L_{\mathrm{loc}}([a, c), E) \rightarrow E, \Delta u(\lambda)=u(\lambda+)-u(\lambda), D: \Lambda \times H L_{\mathrm{loc}}([a, c), E) \rightarrow E$, and $\Lambda$ is a well-ordered subset of $[a, c)$ with $a=\min \Lambda$ and $c=\sup \Lambda$. When $t \in[a, c)$, we denote $\Lambda^{<t}=\{\lambda \in \Lambda: \lambda<t\}$. If a family $(x(\lambda))_{\lambda \in \Lambda}$ of $E$ is locally summable, and $t \in[a, c)$, denote by $\sum_{\lambda \in \Lambda^{<t}} x(\lambda)$ the sum of the family $(x(\lambda))_{\lambda \in \Lambda^{<t}}$.

We say that $u:[a, c) \rightarrow E$ is a solution of problem (5.1) if it satisfies the equations of (5.1), and if it belongs to the set

$$
V=\left\{u \in H L_{\mathrm{loc}}([a, c), E) \mid u \text { is a.e. differentiable and right continuous }\right\} \text {. }
$$

The following result allows us to transform problem (5.1) into an integral equation.

Lemma 5.1 Let $g \in H L_{\mathrm{loc}}([a, c), E)$ and assume that a family $(z(\lambda))_{\lambda \in \Lambda}$ of $E$ is locally summable. Then the problem

$$
u^{\prime}(t)=g(t) \quad \text { a.e. on }[a, c), \quad \Delta u(\lambda)=z(\lambda), \quad \lambda \in \Lambda,
$$

has a unique solution $u$. This solution can be represented as

$$
u(t)=\sum_{\lambda \in \Lambda^{<t}} z(\lambda)+\int_{a}^{t} g(s) d s, \quad t \in[a, c) .
$$

Moreover, $u$ is increasing with respect to $g$ and $z$.

Proof Let $u:[a, c) \rightarrow E$ be defined by (5.3). It is easy to verify that

$$
u^{\prime}(t)=g(t) \text { for a.e. } t \in[a, c) .
$$

For each $\lambda \in \Lambda$ the open interval $(\lambda, S(\lambda))$ does not contain any point of $\Lambda$, so that

$$
\Delta u(\lambda)=u(\lambda+)-u(\lambda)=\lim _{t \rightarrow \lambda+}\left(z(\lambda)+\int_{\lambda}^{t} g(s) d s\right)=z(\lambda), \quad \lambda \in \Lambda .
$$


It follows from (5.3) that

$$
u(t)=u_{1}(t)+u_{2}(t)
$$

where

$$
u_{1}(t)=\int_{a}^{t} g(s) d s, \quad u_{2}(t)=\sum_{\lambda \in \Lambda^{<t}} z(\lambda), \quad t \in[a, c) .
$$

Because $(z(\lambda))_{\lambda \in \Lambda}$ is locally summable, then both $u_{1}$ and $u_{2}$ belong to $V$. This, (5.4) and (5.5) imply that $u$ is a solution of problem (5.2).

If $v \in V$ is a solution of (5.2), then $w=u-v$ belongs to $V$, and $\Delta w(\lambda)=0$ for each $\lambda \in \Lambda$, whence $w$ is a solution of the initial value of problem

$$
w^{\prime}(t)=0 \quad \text { a.e. on }[a, c), \quad w(a)=0
$$

This implies that $w(t) \equiv 0$, i.e., $u=v$.

The last assertion of the lemma is a direct consequence from the representation (5.3) and [10, Lemma 9.11].

Assume that $g \in H L_{\mathrm{loc}}([a, c), E)$ is right regulated. Given $\epsilon>0$ and $b \in(a, c)$, let $\Lambda_{\epsilon}$ be the well ordered subset of $[a, b]$ defined by (2.6), and let $g_{\epsilon}:[a, b] \rightarrow E$ be defined by (4.1). Because $g$ is locally HL integrable, it follows from Proposition 4.1 that $g_{\epsilon}$ is HL integrable on $[a, b]$, and that the family $((S(\alpha)-\alpha) g(\alpha+))_{\alpha \in \Lambda_{\epsilon}^{<b}}$ is summable. Let $\sigma_{\epsilon}(\gamma)$ denote the sum

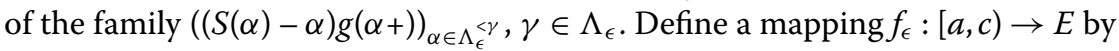

$$
f_{\epsilon}(t)=\sigma_{\epsilon}(\gamma)+(t-\gamma) g(\gamma+), \quad t \in[\gamma, S(\gamma)), \gamma \in \Lambda_{\epsilon}^{<c}
$$

By the proof of Proposition 3.1, $f_{\epsilon}$ is a CD primitive of $g_{\epsilon}$. It follows from Lemma 2.5 that $\left\|g_{\epsilon}(t)-g(t)\right\| \leq \epsilon$ for all $t \in[a, b] \backslash \Lambda_{\epsilon}$. Thus,

$$
\left\|\int_{a}^{t} g(s) d s-\int_{a}^{t} g_{\epsilon}(s) d s\right\|=\left\|\int_{a}^{t} g(s) d s-f_{\epsilon}(t)\right\| \leq \epsilon(t-a) .
$$

The above considerations and Theorem 4.1 imply the following results for solutions of problem (5.2).

Proposition 5.1 Assume that $g \in H L_{\mathrm{loc}}([a, c), E)$ is right regulated, and that the family $(z(\lambda))_{\lambda \in \Lambda}$ is summable. Then for all fixed $b \in(a, c)$ and $\epsilon>0$ the mapping $u_{\epsilon}:[a, b] \rightarrow E$, defined by

$$
u_{\epsilon}(t)=\sum_{\lambda \in \Lambda^{<t}} z(\lambda)+f_{\epsilon}(t), \quad t \in[a, b]
$$

approximates the solution of problem (5.2) uniformly on $[a, b]$ within the accuracy $\epsilon(b-a)$. The differential equation of (5.2) holds in the complement of a countable subset of $[a, b]$. 
In what follows, we assume that $E$ is a Banach space ordered by a regular order cone, and that the function space $H L([a, b], E)$ is ordered a.e. pointwise. The following fixed-point result is a consequence of [10, Theorem 2.16 and Proposition 9.39].

Theorem 5.1 Let $\left[w_{-}, w_{+}\right]=\left\{g \in H L_{\mathrm{loc}}([a, c), E) \mid w_{-} \leq g \leq w_{+}\right\}$be a nonempty order interval in $H L_{\mathrm{loc}}([a, c), E)$. Then every increasing mapping $G: H L_{\mathrm{loc}}([a, b), E) \rightarrow\left[w_{-}, w_{+}\right]$has the smallest and greatest fixed points, and they are increasing with respect to $G$.

Let us impose the following hypotheses on the mappings $f$ and $D$ in problem (5.1).

(f0) There exist locally HL integrable mappings $f_{ \pm}:[a, c) \rightarrow E$ such that $f_{-}(t) \leq f(t, u) \leq f_{+}(t)$ for a.e. $t \in[a, c)$ and for all $u \in H L_{\mathrm{loc}}([a, c), E)$.

(f1) The mapping $f(\cdot, u)$ is right regulated for each $u \in H L_{\mathrm{loc}}([a, c), E)$.

(f2) $f(s, \cdot)$ is increasing for a.e. $s \in[a, c)$.

(D0) $D(\lambda, \cdot)$ is increasing for all $\lambda \in \Lambda$, and there exist $z_{ \pm}: \Lambda \rightarrow E$ such that $z_{-}(\lambda) \leq D(\lambda, u) \leq z_{+}(\lambda)$ for all $\lambda \in \Lambda$ and $u \in H L_{\text {loc }}([a, c), E)$, and that the families $\left(z_{ \pm}(\lambda)\right)_{\lambda \in \Lambda}$ are locally summable.

As an application of Theorem 5.1, we get the following existence and comparison result for problem (5.1).

Theorem 5.2 Let the mappings $f$ and D in (5.1) satisfy the hypotheses (f0) to (f2) and (D0). Then problem (5.1) has the smallest and greatest solutions $u_{*}$ and $u^{*}$ in $V$. Moreover, these solutions are increasing with respect to $D$ and $f$, and they satisfy the differential equation of (5.1) the complement of a countable subset of $[a, c)$.

Proof The hypotheses (f0) and (D0) ensure that the equations

$$
w_{ \pm}(t)=\sum_{\lambda \in \Lambda^{<t}} z_{ \pm}(\lambda)+\int_{a}^{t} f_{ \pm}(s) d s,
$$

define mappings $w_{ \pm} \in H L_{\mathrm{loc}}([a, c), E)$. By using the hypotheses, and [10, Lemma 9.11 and Proposition 9.14], it can be shown that the equation

$$
G u(t):=\sum_{\lambda \in \Lambda^{<t}} D(\lambda, u)+\int_{a}^{t} f(s, u) d s, \quad t \in[a, c)
$$

defines an increasing mapping $G: H L_{\mathrm{loc}}([a, c), E) \rightarrow[w-, w+]$. From Theorem 5.1, it then follows that $G$ has the smallest and greatest fixed points $u_{*}$ and $u^{*}$, and they are increasing with respect to $D$ and $f$. Because by Lemma 5.1 the solutions of problem (5.1) are the same as the fixed points of $G$, then $u_{*}$ and $u^{*}$ are the smallest and greatest solutions of problem (5.1), and they are increasing with respect to $D$ and $f$. To show the validity of the last conclusion, let $u$ be any fixed point of $G$, i.e.,

$$
u(t)=\sum_{\lambda \in \Lambda^{\prec t}} D(\lambda, u)+\int_{a}^{t} f(s, u) d s, \quad t \in[a, c) .
$$

The mapping $f(\cdot, u)$ is by the hypothesis (f1) right regulated, and also locally HL integrable on $[a, c)$. Thus, it has by Theorem 4.1 a CD primitive $\tilde{f}$, and $\int_{a}^{t} f(s, u) d s=\tilde{f}(t)-\tilde{f}(a), t \in$ 
$[a, c)$. Hence there is a countable subset $Z_{1}$ of $[a, c)$ such that $\tilde{f}^{\prime}(t)=f(t, u)$ for each $t \in$ $[a, c) \backslash Z_{1}$. Denoting $Z=Z_{1} \cup \Lambda$, it then follows from (5.12) that

$$
u^{\prime}(t)=\frac{d}{d t}\left(\sum_{\lambda \in \Lambda^{\triangleleft t}} D(\lambda, u)+\int_{a}^{t} f(s, u) d s\right)=f(t, u), \quad t \in[a, c) \backslash Z .
$$

This proves the last conclusion.

Example 5.1 The cone of those elements of $E=c_{0}$ with nonnegative coordinates is regular. Choose $[a, c)=[0, \infty)=\mathbb{R}_{+}$. Let $g_{0}: \mathbb{R}_{+} \rightarrow c_{0}$ be defined by (4.5), and define $q_{i}: \mathbb{R} \rightarrow \mathbb{R}$, $i=1,2, \ldots$, by

$$
q_{i}(s)=\frac{1}{2^{i}} \sum_{m=1}^{i} \sum_{k=1}^{\infty} \frac{\frac{\pi}{2}+\arctan \left(k^{\frac{1}{m}} s\right)}{(k m)^{2}}, \quad s \in \mathbb{R}, i=1,2, \ldots
$$

For $x=\left(x_{1}, x_{2}, \ldots\right) \in c_{0}$, define

$$
g(t, x)=g_{0}(t)+\left(q_{i}\left(\sum_{j=1}^{i} x_{j}\right)\right)_{i=1}^{\infty}, \quad t \in \mathbb{R}_{+} .
$$

Then one can easily verify that $f(t, u)=g(t, u(t))$ satisfies hypotheses (f0) to (f2).

Let $\Lambda$ be a well-ordered subset of real numbers with $\min \Lambda=0$ and $\sup \Lambda=\infty$. Denoting

$$
c(\lambda)=\left(c_{1}(\lambda), c_{2}(\lambda), \ldots\right), \quad \text { where } c_{i}(\lambda)=2^{-i} z_{\lambda}, \lambda \in \Lambda, i=1,2, \ldots
$$

Assuming that the family $\sum_{\lambda \in \Lambda} z_{\lambda}$ is a summable family of real numbers $z_{\lambda}$, then the family $\sum_{\lambda \in \Lambda} c(\lambda)$ is summable in $c_{0}$. Thus, the mapping $D(\cdot, u) \equiv c$ has the properties assumed in (D0). With $c$ and $g$ defined before, consider the problem

$$
u^{\prime}(t)=g(t, u(t)) \quad \text { a.e. on } \mathbb{R}_{+}, \quad \Delta u(\lambda)=c(\lambda), \quad \lambda \in \Lambda .
$$

The above proof shows that the hypotheses of Theorem 5.2 are valid, when $f(t, u)=$ $g(t, u(t))$ and $D(\lambda, u)=c(\lambda)$. Thus, problem (5.13) has the smallest and greatest solutions.

...the infinite series can be totaled at any given point, and this total (more properly, a subtotal) provides the fullness of the sweetness of goal attainment for a given person at a given time and status. But sooner or later, this same person begins to hunger and yearn for new and greater goals, and such adventures in growth will be forever forthcoming in the fullness of time and the cycles of eternity'. ([The Book of Urantia, 118,0,11].) 


\section{Acknowledgements}

The author thanks a referee for very valuable comments.

Received: 25 October 2012 Accepted: 22 March 2013 Published: 15 April 2013

\section{References}

1. Dieudonné, J: Foundations of Modern Analysis. Academic Press, New York (1960)

2. Heikkilä, S, Lakshmikantham, V: Monotone Iterative Techniques for Discontinuous Nonlinear Differential Equations. Dekker, New York (1994)

3. Bourbaki, N: General Topology, Part I. Hermann, Paris (1968)

4. Bourbaki, N: General Topology, Part II. Hermann, Paris (1968)

5. Di Piazza, L, Marraffa, V: The McShane, PU and Henstock integrals of Banach valued functions. Czechoslov. Math. J. 52, 609-633 (2002)

6. Schwabik, Š, Guoju, Ye: Topics in Banach Space Integration. World Scientific, Singapore (2005)

7. Swartz, C: Gauge integrals and series. Math. Bohem. 129(3), 324-332 (2004)

8. Series (mathematics), Wikipedia, the free encyclopedia

9. Schechter, E: Handbook of Analysis and Its Foundations. Academic Press, San Diego (1997)

10. Carl, S, Heikkilä, S: Fixed Point Theory in Ordered Spaces and Applications. Springer, Berlin (2011)

11. Boccuto, A, Sambucini, AR: The Henstock-Kurzweil integral for functions defined on unbounded intervals and with values in Banach spaces. Acta Math. 7, 3-17 (2004)

12. Marraffa, V: Strongly measurable Kurzweil-Henstock type integrable functions and series. Quaest. Math. 31(4), 379-386 (2008)

13. Faure, C-A, Mawhin, J: Integration over unbounded multidimensional intervals. J. Math. Anal. Appl. 205(1), 65-77 (1997)

14. Lindelöf, E: Differential and Integral Calculus and its Applications. (III,2), Helsinki (1940) (in Finnish)

doi:10.1186/1687-2770-2013-91

Cite this article as: Heikkilä: On summability, integrability and impulsive differential equations in Banach spaces. Boundary Value Problems 2013 2013:91.

\section{Submit your manuscript to a SpringerOpen ${ }^{0}$ journal and benefit from:}

- Convenient online submission

- Rigorous peer review

- Immediate publication on acceptance

- Open access: articles freely available online

- High visibility within the field

- Retaining the copyright to your article 